\title{
Evaluation of the ESGE recommendations for COVID-19 pre-endoscopy risk-stratification in a high-volume center in Germany
}

\section{(ㄷ)(i) $\odot$}

Authors

Stephan Zellmer*, 1, Alanna Ebigbo*, 1, Maria Kahn', Anna Muzalyova', Johanna Classen', Vivian Grünherz', Selin Temizel ${ }^{2}$, Christine Dhillon ${ }^{3}$, Helmut Messmann¹, Christoph Römmele ${ }^{1,3}$

Institutions

1 Department of Gastroenterology and Infectious Diseases, University Hospital of Augsburg, Augsburg, Germany

2 Department of Hygiene and Environmental Medicine, University Hospital of Augsburg, Augsburg, Germany

3 Covid-19 Task Force, University Hospital of Augsburg, Augsburg, Germany

submitted 11.3.2021

accepted after revision 25.5.2021

Bibliography

Endosc Int Open 2021; 09: E1556-E1560

DOI 10.1055/a-1526-1169

ISSN 2364-3722

(C) 2021. The Author(s).

This is an open access article published by Thieme under the terms of the Creative Commons Attribution-NonDerivative-NonCommercial License, permitting copying and reproduction so long as the original work is given appropriate credit. Contents may not be used for commercial purposes, or adapted, remixed, transformed or built upon. (https://creativecommons.org/licenses/by-nc-nd/4.0/)

Georg Thieme Verlag KG, Rüdigerstraße 14,

70469 Stuttgart, Germany

Corresponding author

Stephan Zellmer, III. Medizinische Klinik, Universitätsklinikum

Augsburg, Stenglinstraße 2, 86156 Augsburg, Germany

stephan.zellmer@uk-augsburg.de

Supplementary material is available under

https://doi.org/10.1055/a-1526-1169

\section{ABSTRACT}

Background and study aims The European Society of Gastrointestinal Endoscopy (ESGE) has defined COVID-19 infection prevention and control strategies within the endoscopy unit. These include pre-endoscopic questionnaire-based risk-stratification as well as pre-procedure viral testing. Real-life data on the effectiveness of these measures are presented here.

Patients and methods Data from the outpatient endoscopic unit of the University Hospital Augsburg between July 1, 2020 and December 31, 2020 including the second pandemic wave were reviewed retrospectively. All patients were assessed with a pre-endoscopic risk-stratification questionnaire as well as viral testing using an antigen point-of-care test $(\mathrm{Ag}-\mathrm{POCT})$ in conjunction with a standard polymerase chain reaction (PCR) test. Highly elective procedures were postponed. The theoretically expected number of SARS-CoV-2-positive patients was simulated and compared with the actual number. In addition, endoscopy staff was evaluated with a rapid antibody test to determine the number of infections among the personnel.

Results In total, 1029 procedures, 591 questionnaires, 591 Ag-POCTs, and 529 standard PCR tests were performed in 591 patients. 247 procedures in 142 patients were postponed. One Ag-POCT was positive but with a negative PCR test, while one PCR test was positive but with a negative AgPOCT. This was lower than the theoretically expected number of COVID-19-positive patients $(n=15)$. One of $43 \mathrm{em}$ ployees $(2.3 \%)$ in the outpatient endoscopy unit was seropositive.

Conclusions Pre-endoscopic risk management including questionnaire-based risk stratification and viral testing seems to be an effective tool in combination with personal protective equipment for SARS-CoV-2 infection prevention and control within the endoscopy unit even in a high-prevalence setting.

\footnotetext{
* These authors contributed equally.
} 


\section{Introduction}

The rapid spread of coronavirus disease 2019 (COVID-19) and its debilitating impact on health systems has led to a global health crisis of unprecedented proportions. Health care workers (HCW) exposed to aerosols and tracheobronchial droplets during gastrointestinal endoscopy are at a higher risk of infection [1]. In addition, severe acute respiratory syndrome coronavirus-2 (SARS-CoV-2) has been isolated in the feces of patients with COVID-19, raising the question of a possible fecal-oral route of transmission [2].

For these reasons, pre-procedure screening of patients presenting for endoscopy has been suggested in various recommendations [3-5]. Routine pre-endoscopic screening could have enormous advantages for infection prevention and control in endoscopic departments. The European Society of Gastrointestinal Endoscopy (ESGE) position statements recommend pre-endoscopic risk stratification by questioning for symptoms and positive history one day before and on the day of endoscopy [3]. Furthermore, it is recommended that highly elective examinations may temporarily be postponed. A key point of the recommended screening strategy is strict viral testing using molecular diagnostic tests, such as polymerase chain reaction (PCR), if available. Besides standard PCR, widely used rapid antigen tests are available for the detection of SARSCoV-2 infection. These tests can be performed as an antigen point-of-care test (Ag-POCT) in the immediate proximity of the endoscopy unit. However, the position paper does not recommend Ag-POCTs due to insufficient data [6].

In the endoscopy unit of Augsburg University Hospital, preprocedure risk stratification was performed according to the recommendations in the ESGE position statement using a twostage pre-procedure questionnaire 1 to 2 days prior to a patient's appointment as well as on the day of their presentation to the endoscopy unit. In addition, especially during the peak of the second wave in November and December 2020 (Supplement 1), highly elective examinations such as surveillance of post-polypectomy or post-surgery colorectal cancer patients were postponed. Besides the standard PCR test, Ag-POCTs were performed in all patients to generate real-life data for a prospective evaluation of this test method.

Pre-endoscopic screening procedures are resource-sensitive and cost-intensive [6]; in addition, data on the effectiveness of these measures in endoscopic facilities are lacking. In this study, data on the effectiveness of pre-endoscopic risk management and testing recommended by ESGE in a high-volume endoscopic center in Germany during the second wave of the COVID-19 pandemic are presented.

\section{Patients and methods}

\section{Patient screening}

We analyzed all outpatients who presented to the endoscopy unit of the Augsburg University Hospital between 01.07.2020 and 31.12.2020.

Pre-endoscopic risk management was performed according to the recommendations in the ESGE position statements [3, 7].
For this purpose, patients were screened for COVID-19 using a two-stage pre-procedure questionnaire for risk stratification, 1 to 2 days prior to their appointment as well as on the day of their presentation to the endoscopy unit (Supplement 2). After questionnaire-based risk stratification, patients were tested with an antigen-POCT (standard F Covid19 Ag FIA/SD; Co. Biosensor). In addition, most patients also underwent a standard PCR test (see supplemental material). A flowchart showing the exact procedure in our endoscopy unit is demonstrated in

- Fig. 1. For the antigen-POCT and the standard PCR test, nasopharyngeal or oropharyngeal swabs were taken by appropriately trained staff members in a separate room of the endoscopy unit on the day of the procedure. The study was conducted in accordance with the Declaration of Helsinki and Good Clinical Practice guidelines. Ethics approval was granted by the Ethics committee of the Ludwig-Maximilians-Universität München (EK-Nr. 20-1052).

\section{Simulation of expected COVID-19 case numbers and number of cancelled or postponed procedures}

The catchment area of Augsburg University Hospital was defined using controlling data from previous years. The prevalence in the population in the given catchment area was carried out following Sultan et al. (2020), and a theoretically expected number of cases of SARS-CoV-2 positive patients was calculated as follow [8]:

1. The cumulative number of cases was provided by the Bavarian State Office for Health and Food Safety for each day of the study period.

2. The number of new cases was calculated as a difference between the cumulative number of cases on the previous day and the cumulative number of cases on the current / considered day,

3. Using the daily data from the previous step, the cumulative number of active cases for the past 14 days was calculated.

4. In line with Forde et al. [9] and Benatia et al. [10], the number of active cases was adjusted for the SARS-CoV-2 positive population in the catchment area by multiplying by 10 .

5. The population in the catchment area was determined using information provided by the Bavarian State Office for Health and Food Safety.

6. The number of expected SARS-CoV-2-positive patients was determined by dividing the adjusted number of active cases within the population in the catchment area and multiplying by the number of patients regularly scheduled in the outpatient's endoscopy unit within the study period.

To obtain the reduction in total case load and an estimate for the number of examinations cancelled or postponed, we compared the case numbers for the study period with those for the previous year.

\section{Employee screening}

We retrospectively evaluated rapid antibody tests (NADAL COVID-19 $\mathrm{lgG} / \mathrm{lgM}$ ) performed in the last week of the study period among employees and staff of the endoscopic outpatient department. 


\section{Strategy}

\section{Before the appointment}

Patients are contacted by telephone one to two days prior to presentation to our clinic. A risk-stratification questionnaire is completed via telephone

No contraindication for presentation

\section{Patient presenting in the endoscopy unit}

Completion of the questionnaire on symptoms / recent contacts to COVID-19 infected persons / current quarantine/ stay abroad

$\downarrow \begin{aligned} & \text { No contraindication } \\ & \text { for presentation }\end{aligned}$

POCT-antigen test (result within 20 minutes) + standard-PCR test (result the following day)

Negative POCT result

In high prevalence phase of the pandemic, performance of the endoscopy with high-risk PPE

Contraindication for presentation

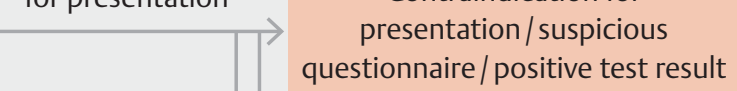

Contraindication

for presentation

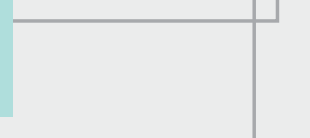

Positive test result positve PCR test after 24 hours
Elective procedure

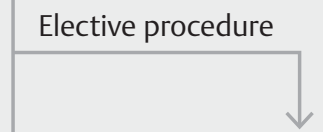

Re-appoint after quarantine/clarification etc.

Medically urgent procedure
Performance of the endoscopy with high-risk PPE and measures following the COVID-19 requirements

\section{Post-contact tracing}

- Contact the patient

- Retrace all contacts the patient had within the hospital

- Inform the public health agency

- Fig. 1 Screening strategy in the endoscopy unit for outpatients. Ag-POCT, antigen point-of-care test; COVID-19, corona virus disease 2019; PCR, polymerase chain reaction; PPE, personal protective equipment; SARS-CoV-2, severe acute respiratory syndrome coronavirus 2.

\section{Results}

\section{Examination volume and pre-endoscopic risk management}

The expected examination volume between July and December 2020 estimated using prior-year data was 1276 procedures in 733 patients ( $\mathbf{F i g . 2}$ ). A total of 238 procedures in 137 patients $(18.7 \%)$ were cancelled between July and December 2020, of which, 185 procedures in 106 patients were cancelled or postponed during the peak of the second wave of the pandemic in November and December.

Examinations and procedures were cancelled or postponed due to COVID-19-like symptoms, a positive history in the preendoscopic screening questionnaire, or due to a highly elective indication. A total of 596 patients (81.3\%) with 1038 planned procedures presented to the endoscopy unit and underwent pre-endoscopic risk stratification in the endoscopy unit. Five patients $(1.0 \%)$ were sent home without further testing or intervention on the day of pre-procedure screening due to highrisk stratification according to the questionnaire. Two of them had recently travelled to a high-risk area and three of them had COVID-19-related symptoms.
A total of 591 patients were tested with the Ag-POCT, of which, $529(89.5 \%)$ received a standard PCR test in parallel. The antigen test provided a result within 20 minutes, while the results of the standard PCR test were available with a time delay of up to 24 hours.

Of the 1029 procedures performed, 636 (61.8\%) were endoscopies of the upper gastrointestinal tract (543 gastroscopies, 1 endoscopic retrograde cholangiopancreatography, 92 endoscopic ultrasounds).

\section{Results of the Ag-POCT}

Ag-POCT was positive in one patient $(0.2 \%)$ but with a negative standard PCR test while one other patient tested positive with standard PCR test but negative in the Ag-POCT. However, this patient had a high cycle threshold (CT) value of 35.6.

\section{Expected COVID-19 case number}

The simulations were performed as described in the methods section. The expected case number of SARS-CoV-2 positive patients for the study period was 15 . 


\section{3 patients with 1276 endoscopic procedures}

Based on the ESGE position statement [3, 7], 238 procedures in 137 patients were cancelled or postponed due to symptoms or a positive history in the pre-endoscopy screening questionnaire, by

596 (81\%) patients with 1038 planned procedures presenting in the endoscopy unit<smiles>C=CC</smiles>

Completion of the questionnaire on symptoms/recent contacts to SARS-CoV-2 infected persons/current

5 (1\%) patients were excluded from

9 procedures due to COVID-19 related

symptoms or positive history

591 (99\%) patients with planned endoscopic procedures<smiles>C1=CC=C1</smiles>

591 (100\%) patients underwent Ag-POCT + 529 (90\%) underwent standard PCR test

1 positive $\mathrm{Ag}-\mathrm{POCT}$ that was interpreted

false positive based on the patient's history

1 positive standard PCR test

(result received after the procedure)

591 (100\%) patients with 1029 performed endoscopic procedures

Fig. 2 Screening data for the study period. Ag-POCT, antigen point-of-care-test; COVID 19, corona virus disease 2019 ESGE, European Society of Gastrointestinal Endoscopy; PCR, polymerase chain reaction; SARS-CoV-2, severe acute respiratory syndrome coronavirus 2 .

\section{Employee screening}

Fourty-three employees/health care workers in the endoscopy department underwent an antibody rapid test. One endoscopy nurse had a positive result while 42 were negative. All workers were asymptomatic on the day of the test.

\section{Discussion}

In this paper, we present real-life data on the effectiveness of pre-endoscopic risk management according to the recommendations of the ESGE in a high-volume endoscopic center in Germany during the second wave of the COVID-19 pandemic.

The study was conducted in Augsburg which has been a local hotspot during the ongoing second wave of the pandemic. Seven-day incidence rates as high as 400 per 100,000 were reported for the region [11].
The calculated number of expected SARS-CoV-2-positive patients, taking into account the daily prevalence in the catchment area, was 15 . This number is in contrast to the one patient who tested positive via PCR test. We believe the reason for the low number of detected cases was the strict questionnairebased risk stratification performed via telephone, 1 to 2 days prior to the intervention, as well as on the day of the intervention. It is also possible that the temporary postponement of highly elective procedures may have resulted in a decreased number of SARS-CoV-2-positive patients presenting to the endoscopy unit. According to the simulation based on the prevalence and incidence rates in the region, about 14 additional patients with COVID-19 should have presented to the unit. However, the estimated number of unreported cases in Germany seems to be overestimated with a factor of 10 . Other sources suggest a factor of three to five $[12,13]$. In this case, however, there would still have been an expected five to eight patients instead with a SARS-CoV-2 infection.

With only one positive PCR test result and one positive result in the $\mathrm{Ag}-\mathrm{POCT}$, it is not possible to correctly estimate the sensitivity and specificity of the Ag-POCT. The patient who tested positive in the Ag-POCT had a negative PCR test. The patient had previously been tested positive several times in the AgPOCT. However, the PCR test had always been negative and there was no history of previous disease. For this reason, we assume the Ag POCT to have been false positive in this case presumably due to bacterial colonization [14]. In the PCR test-positive patient, the CT value was 35.6, which implies a low pharyngeal viral load [15]. Because the PCR test result was received about 24 hours later, all health care workers involved in the direct care of this patient underwent clinical follow-up and testing but remained negative and asymptomatic. After receiving the positive PCR test result, the patient was sent to home isolation according to the orders of the local health department. However, the patient did not have any COVID-19 typical symptoms at any time.

A further indication for the effectiveness of the risk-management measures is the low number of infected employees within the unit. Only one endoscopy worker (2.3\%) had a serologically detectable history of past infection, which had been acquired within the private family environment. Of course, all recommended personal protective equipment (PPE) standards were used routinely for all endoscopy procedures irrespective of the pre-endoscopy risk-stratification.

Our findings are in line with publications by Recipi et al. and Hayee et al., who described the use of PPE and reduction of elective procedures as a useful tool for SARS-CoV-2 infection prevention $[16,17]$.

The Ag-POCT, which was part of our study evaluation, is cheap, widely available, easily performable at the point-ofcare, and produces results within 20 minutes [18]. However, real-life data on test accuracy in endoscopy units are lacking.

Nevertheless, Ag-POCT may be considered in combination with questionnaire-based risk-stratification and use of the recommended PPE for units without access to the resource-intensive and significantly more expensive molecular diagnostic PCR test. The approval of COVID-19 vaccines in the last few months, 
and the widespread vaccination of endoscopy health care workers, may require an adjustment of the recommendations presented in this study.

The major limitation of this study is the retrospective evaluation, which made it difficult to evaluate the exact number of patients whose procedures were cancelled specifically due to a high-risk stratification via telephone. However, most elective endoscopic examinations were continued during the second wave of the pandemic, which means that a greater number of cancelled or postponed procedures can be attributed to a high-risk stratification according to the questionnaire.

A further limitation is the low number of SARS-CoV-2-positive PCR test results $(n=1)$ produced during the study period, making the planned direct prospective comparison between the Ag-POCT and PCR tests impossible. However, and as described above, the low number of positive patients can be attributed to the risk-stratification approach described above.

\section{Conclusions}

In conclusion, pre-endoscopic risk management recommended by ESGE, especially questionnaire-based risk stratification in conjunction with viral testing, seems to be an effective tool in combination with PPE for SARS-CoV-2 infection prevention and control within the endoscopy unit even in a high-prevalence setting.

\section{Competing interests}

The authors declare that they have no conflict of interest.

\section{References}

[1] Repici A, Maselli R, Colombo M et al. Coronavirus (COVID-19) outbreak: what the department of endoscopy should know. Gastrointest Endosc 2020; 92: 192-197

[2] Gu J, Han B, Wang J. COVID-19: Gastrointestinal manifestations and potential fecal-oral transmission. Gastroenterology 2020; 158: 15181519

[3] Gralnek IM, Hassan C, Beilenhoff U et al. ESGE and ESGENA Position Statement on gastrointestinal endoscopy and the COVID-19 pandemic. Endoscopy 2020; 52: 483-490
[4] Hennessy B, Vicari J, Bernstein B et al. Guidance for resuming GI endoscopy and practice operations after the COVID-19 pandemic. Gastrointest Endosc 2020; 92: 743-747 e741

[5] Sultan S, Altayar O, Siddique SM et al. AGA Institute Rapid review of the gastrointestinal and liver manifestations of COVID-19, meta-analysis of international data, and recommendations for the consultative management of patients with COVID-19. Gastroenterology 2020; 159: 320-334 e327

[6] Ebigbo A, Rommele C, Bartenschlager C et al. Cost-effectiveness analysis of SARS-CoV-2 infection prevention strategies including preendoscopic virus testing and use of high risk personal protective equipment. Endoscopy 2021; 53: 156-161

[7] Gralnek IM, Hassan C, Beilenhoff U et al. ESGE and ESGENA Position Statement on gastrointestinal endoscopy and COVID-19: An update on guidance during the post-lockdown phase and selected results from a membership survey. Endoscopy 2020; 52: 891-898

[8] Sultan S, Siddique SM, Altayar O et al. AGA Institute rapid review and recommendations on the role of pre-procedure SARS-CoV-2 testing and endoscopy. Gastroenterology 2020; 159: 1935-1948 e1935

[9] Forde J], Goldberg D, Sussman D et al. Yield and implications of preprocedural COVID-19 polymerase chain reaction testing on routine endoscopic practice. Gastroenterology 2020; 159: 1538-1540

[10] Benatia D, Godefroy R, Lewis ]. Estimating COVID-19 prevalence in the United States: a sample selection model approach. medRxiv 2020: doi:10.1101/2020.04.20.20072942

[11] Stadt Augsburg. Coronavirus - Fallzahlen Augsburg [cited 8 March 2021]. 2021: Available from: https://www.augsburg.de/umweltsoziales/gesundheit/coronavirus/fallzahlen

[12] Pritsch M, Radon K, Bakuli A et al. Prevalence and Risk factors of infection in the representative COVID-19 cohort Munich. Preprint 2020. doi: $10.2139 /$ ssrn. 3745128

[13] DunklezifferRadar e.V. Covid-19 Dunkelzifferradar. 2021: Available from: (access date 25.02.2021) https://covid19.dunkelzifferradar.de

[14] Cronin UP, Girardeaux L, O'Meara E et al. Protein A-Mediated binding of staphylococcus spp. to antibodies in flow cytometric assays and reduction of this binding by using Fc receptor blocking reagent. Appl Environ Microbiol 2020; 86: doi:10.1128/AEM.01435-20

[15] Tom MR, Mina MJ. To interpret the SARS-CoV-2 Test, consider the cycle threshold value. Clin Infect Dis 2020; 71: 2252-2254

[16] Repici A, Aragona G, Cengia G et al. Low risk of COVID-19 transmission in Gl endoscopy. Gut 2020; 69: 1925-1927

[17] Hayee B, East J, Rees C] et al. Multicentre prospective study of COVID19 transmission following outpatient $\mathrm{Gl}$ endoscopy in the UK. Gut 2021; 70: 825-828

[18] Perez-Garcia F, Romanyk J, Gomez-Herruz P et al. Diagnostic performance of CerTest and Panbio antigen rapid diagnostic tests to diagnose SARS-CoV-2 infection. J Clin Virol 2021; 137: 104781 